Frontiers in Heat and Mass Transfer

\title{
NUMERICAL SOLUTION ON HEAT TRANSFER MAGNETOHYDRODYNAMIC FLOW OF MICROPOLAR CASSON FLUID OVER A HORIZONTAL CIRCULAR CYLINDER WITH THERMAL RADIATION
}

\author{
Hamzeh T. Alkasasbeh* \\ Department of Mathematics, Faculty of Science, Ajloun National University, P.O.Box 43, Ajloun 26810, Jordan
}

\begin{abstract}
This paper focuses on the numerical solution for magnetohydrodynamic (MHD) flow of micropolar Casson fluid with thermal radiation over a horizontal circular cylinder. The nonlinear partial differential equations of the boundary layer are first transformed into a non-dimensional form and then solved numerically using an implicit finite difference scheme known as Keller-box method. The The effects of the emerging parameters, namely Casson fluid parameter, magnetic parameter, radiation parameter and micropolar parameter on the local Nusselt number and the local skin friction coefficient, as well as the temperature, velocity and angular velocity profiles are shown graphically and discussed. The present results of local Nusselt number and the local skin friction for viscous fluid are found to be in good agreement with the literature.
\end{abstract}

Keywords: Casson Fluid; Horizontal Circular Cylinder; Magnetohydrodynamic (MHD); Micropolar Fluid; Numerical Solution; Radiation.

\section{INTRODUCTION}

Casson fluids in the presence of heat transfer is widely used in the processing of chocolate, foams, syrups, nail, toffee and many other foodstuffs (Ramachandra et al. (2013). Casson (1959), in his pioneering work introduced this model to simulate industrial inks. Later on, a substantial study has been done on the Casson fluid flow because of its important engineering applications. Mustafa et al. (2011) have studied the heat transfer flow of a Casson fluid over an impulsive motion of the plate using the homotopy method. The exact solution of forced convection boundary layer Casson fluid flow toward a linearly stretching surface with transpiration effects are reported by Mukhopadhyay et al. (2013). In the same year, Subba et al. (2015) considered the velocity and thermal slip conditions on the laminar boundary layer heat transfer flow of a Casson fluid past a vertical plate. Mahdy and Ahmed (2017) studied the effect of magnetohydrodynamic on a mixed convection boundary flow of an incompressible Casson fluid in the stagnation point of an impulsively rotating sphere. The convective boundary layer flow of Casson nanofluid from an isothermal sphere surface is presented by Nagendra et al. (2017). Mehmood et al. (2017) investigated the micropolar Casson fluid on mixed convection flow induced by a stretching sheet. Shehzad et al. (2013) discussed the viscous chemical reaction effects on the MHD flow of a Casson fluid over a porous stretching sheet. Recently, Khalid et al. (2015) developed exact solutions for unsteady MHD free convection flow of a Casson fluid past an oscillating plate. Amongst the various investigations on Casson fluid, the reader is referred to some new attempts made in Qasim and Noreen (2014; Hussanan et al. (2014) and Haq et al. (2014), and the references therein.

Among the class of several other non-Newtonian fluid models namely micropolar fluids, this fluid flow lies in the extension of the constituent equation for Newtonian fluid, so that more complex fluids such as liquid crystal, particle suspensions, animal blood, lubrication and turbulent shear flows can be described by this theory Lukaszewicz (1999). The theory of micrpolar fluids was first introduced by Eringen (1966). Ariman et al. (1973) investigated the application of micropolar fluid mechanics as review paper. The recent book by Eringen (2001) presented a useful account of the theory and extensive surveys of literature of micropolar fluid theory.

The study of boundary layer flow on a horizontal circular cylinder was first studied by Blasius (1908), who successfully solved the momentum equation of forced convection boundary layer flow. Merkin (1976) considered the free convection boundary layer on an isothermal horizontal cylinder with constant wall temperature and became the first who obtained the exact solution for this problem. Ingham (1978) developed the numerical method to solved free convective boundary layer flow on an isothermal horizontal cylinder. Merkin and Pop (1988) presented the numerical solution of the free convection boundary layer flow on a horizontal circular cylinder with constant heat flux using the Keller-box method. Next, the extended by the work of Merkin (1976) and Merkin and Pop (1988) for free convection boundary layer flow on a horizontal circular cylinder in viscous fluid to a micropolar fluid was investigated by Nazar et al. (2002). Moreover Salleh and Nazar (2010) and Alkasasbeh et al $(2015 ; 2014)$ work with Newtonian heating. Gaffar et al. (2015) investigated the laminar boundary layer flow and heat transfer of a Tangent Hyperbolic non-Newtonian fluid from horizontal circular cylinder with slip condition. Recently, Gaffar et al. (2017) studied the magnetohydrodynamic (MHD) free convection flow and heat transfer of non-Newtonian tangent hyperbolic fluid from horizontal circular cylinder with convective boundary conditions.

Based on the above contribution, the aim of present study is to investigate the effect of MHD on free convective boundary layer flow about a horizontal circular cylinder in a micropolar Casson fluid with thermal radiation and this problem has to the author knowledge not appeared thus far in the scientific literature.

*Corresponding author Email: hamzahtahak@yahoo.com 


\section{MATHEMATICAL MODELING}

Consider the steady, laminar, two-dimensional, viscous, incompressible, buoyancy-driven convection heat transfer flow from a horizontal permeable circular cylinder embedded in a micropolar Casson fluid. For many actual fluids and flow conditions, a simple and convenient way to express the density difference $\left(\rho-\rho_{\infty}\right)$ in the buoyancy term of the momentum equations is given by the Boussinesq approximation $\rho=\rho_{\infty}\left[1-B\left(T-T_{\infty}\right)\right]$, where $\rho_{\infty}$ is the constant local density, $T$ is the local temperature, $T_{\infty}$ is the temperature of the ambient medium. $\rho$ is the fluid density and $B$ is the thermal expansion coefficient,. Figure 1 shows the flow model and physical coordinate system. The $\bar{x}$-coordinate is measured along the circumference of the horizontal cylinder from the lowest point and the $\bar{y}$-coordinate is measured normal to the surface, with $a$ denoting the radius of the horizontal cylinder. $\bar{x} / a$ is the angle of the $\bar{y}-$ axis with respect to the vertical $0 \leq \bar{x} / a \leq \pi$. The gravitational acceleration, $g$ acts downwards. Both the horizontal cylinder and the fluid are maintained initially at the same temperature. Instantaneously they are raised to a temperature $T_{w}>T_{\infty}$ the ambient temperature of the fluid which remains unchanged.

The constitutive relationship for an incompressible Casson fluid flow, reported by Mukhopadhyay et al. (2013).

$$
\tau_{i j}= \begin{cases}2\left(\mu_{B}+p_{y} \sqrt{2 \pi}\right) e_{i j} & \pi>\pi_{c}, \\ 2\left(\mu_{B}+p_{y} \sqrt{2 \pi}\right) e_{i j} & \pi<\pi_{c},\end{cases}
$$

where $\pi=e_{i j} e_{i j}, e_{i j}$ is the $(i, j)-$ th component of the deformation rate, $\mu_{B}$ is the plastic dynamic viscosity of the non-Newtonian fluid, $\pi_{c}$ is a critical value of this product based on the non-Newtonian model and $p_{y}$ is the yield stress of the fluid.

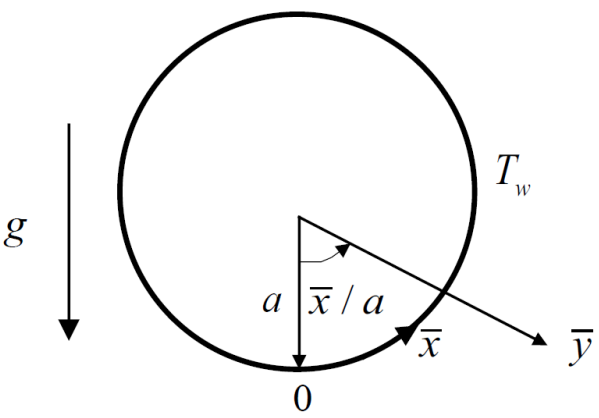

Fig. 1 Physical model and coordinate system

Introducing the boundary layer approximations, the continuity, momentum, microrotation and energy equations, respectively can be written as follows:

$$
\begin{gathered}
\frac{\partial \bar{u}}{\partial \bar{x}}+\frac{\partial \bar{v}}{\partial \bar{y}}=0 \\
\bar{u} \frac{\partial \bar{u}}{\partial \bar{x}}+\bar{v} \frac{\partial \bar{u}}{\partial \bar{y}}=\frac{(\mu+\kappa)}{\rho}\left(1+\frac{1}{\beta}\right) \frac{\partial^{2} \bar{u}}{\partial \bar{y}^{2}}+ \\
g B\left(T-T_{\infty}\right) \sin \left(\frac{\bar{x}}{a}\right)+\frac{\kappa}{\rho} \frac{\partial \bar{H}}{\partial \bar{y}}-\frac{\sigma B^{2}}{\rho} \bar{u} \\
\bar{u} \frac{\partial \bar{H}}{\partial \bar{x}}+\bar{v} \frac{\partial \bar{H}}{\partial \bar{y}}=\frac{\phi}{\rho j} \frac{\partial^{2} \bar{H}}{\partial \bar{y}^{2}}-\frac{\kappa}{\rho j}\left(2 \bar{H}+\frac{\partial \bar{u}}{\partial \bar{y}}\right), \\
\bar{u} \frac{\partial T}{\partial \bar{x}}+\bar{v} \frac{\partial T}{\partial \bar{y}}=\alpha \frac{\partial^{2} T}{\partial \bar{y}^{2}}-\frac{1}{\rho c_{\rho}} \frac{\partial q_{r}}{\partial \bar{y}}
\end{gathered}
$$

these equations are subjected to the boundary conditions Nazar et al. (2002),

$$
\begin{gathered}
\bar{u}=\bar{v}=0, T=T_{w}, \bar{H}=-\frac{1}{2} \frac{\partial \bar{u}}{\partial \bar{y}} \text { as } \bar{y}=0, \\
\bar{u} \rightarrow 0, T \rightarrow T_{\infty}, \quad H \rightarrow 0, \text { as } \bar{y} \rightarrow \infty,
\end{gathered}
$$

where $\bar{u}$ and $\bar{v}$ are the velocity components along the $\bar{x}$ and $\bar{y}$ directions, respectively, $\bar{H}$ is the angular velocity of micropolar fluid, $\kappa$ is the vortex viscosity, $g$ is the gravity acceleration, $k$ is the thermal conductivity, $\sigma$ is the electric conductivity, $\alpha$ is the thermal diffusivity, $v$ is the kinematic viscosity, $\mu$ is the dynamic viscosity, $c_{\rho}$ is the specific heat, $j=a^{2} / \sqrt{G r}$ is the microinertia density, $\beta=\mu_{B} \sqrt{2 \pi_{c}} / p_{y}$ is the parameter of the Casson fluid and the spin gradient viscosity $\phi=(\mu+\kappa / 2) j$. We introduce now the following non-dimensional variables Nazar et al. (2002),

$$
\begin{gathered}
x=\frac{\bar{x}}{a}, \quad y=\frac{\sqrt[4]{G r}}{a} \bar{y}, \quad r=\frac{\bar{r}}{a}, \\
u=\frac{a}{v \sqrt{G r}} \bar{u}, \quad v=\frac{a}{v \sqrt[4]{G r}} \bar{v}, \quad H=\frac{a^{2}}{v \sqrt[4]{G r^{3}}} \bar{H}, \\
\theta=\frac{T-T_{\infty}}{T_{w}-T_{\infty}},
\end{gathered}
$$

where $G r=g B\left(T_{w}-T_{\infty}\right) a^{3} / v^{2}$ is the Grashof number. Using the Rosseland approximation for radiation, the radiative heat flux is simplified as (Bataller (2008))

$$
q_{r}=-\frac{4 \sigma^{*}}{3 k^{*}} \frac{\partial T^{4}}{\partial \bar{y}}
$$

where $\sigma^{*}$ and $k^{*}$ are the Stefan-Boltzmann constant and the mean absorption coefficient, respectively. We assume that the temperature differences within the flow through the micropolar fluid such as that the term $T^{4}$ may be expressed as a linear function of temperature. Hence, expanding $T^{4}$ in a Taylor series about $T_{\infty}$ and neglecting higher-order terms, we get

$$
T^{4} \cong 4 T_{\infty}^{3} T-3 T_{\infty}^{4},
$$

substituting variables (6)-(8) into equations (1)-(4), we obtain the following non-dimensional equations of the problem under consideration:

$$
\begin{gathered}
\frac{\partial u}{\partial x}+\frac{\partial v}{\partial y}=0 \\
u \frac{\partial u}{\partial x}+v \frac{\partial u}{\partial y}=\left(1+K+\frac{1}{\beta}\right) \frac{\partial^{2} u}{\partial y^{2}}+\theta \sin x-M u+K \frac{\partial H}{\partial y} \\
u \frac{\partial H}{\partial x}+v \frac{\partial H}{\partial y}=-K\left(2 H+\frac{\partial u}{\partial y}\right)+\left(1+\frac{K}{2}\right) \frac{\partial^{2} H}{\partial y^{2}} \\
u \frac{\partial \theta}{\partial x}+v \frac{\partial \theta}{\partial y}=\frac{1}{\operatorname{Pr}}\left(1+\frac{4}{3} R\right) \frac{\partial^{2} \theta}{\partial y^{2}}
\end{gathered}
$$

where $K=\kappa / \mu$ is the material or micropolar parameter, $\operatorname{Pr}=v / \alpha$ is the Prandtl number, $M=\sigma B^{2} a^{2} / \nu \rho \sqrt{G r}$ is the magnetic parameter and $R=\alpha k^{*} \rho c_{\rho} / 4 \sigma^{*} T_{\infty}^{3}$ is the radiation parameter.. The boundary conditions (5) become

$$
\begin{gathered}
u=v=0, \quad \theta=1, \quad H=-\frac{1}{2} \frac{\partial u}{\partial y} \text { at } y=0, \\
u \rightarrow 0, \quad \theta \rightarrow 0, \quad H \rightarrow 0 \text { as } y \rightarrow \infty,
\end{gathered}
$$

To solve equations (9) to (12), subjected to the boundary conditions (13), we assume the following variables: 


$$
\psi=x f(x, y), \quad \theta=\theta(x, y), \quad H=x h(x, y),
$$

where $\psi$ is the stream function defined as

$$
u=\frac{\partial \psi}{\partial y} \text { and } v=-\frac{\partial \psi}{\partial x}
$$

so that $u=x \frac{\partial f}{\partial y}$ and $v=-\left(f(x, y)+x \frac{\partial f}{\partial x}\right)$, which satisfies the continuity equation (9). Thus, (10) to (12) become

$$
\begin{array}{r}
\left(1+K+\frac{1}{\beta}\right) \frac{\partial^{3} f}{\partial y^{3}}+f \frac{\partial^{2} f}{\partial y^{2}}-\left(\frac{\partial f}{\partial y}\right)^{2}+\frac{\sin x}{x} \theta- \\
M \frac{\partial f}{\partial y}+K \frac{\partial h}{\partial y}=x\left(\frac{\partial f}{\partial y} \frac{\partial^{2} f}{\partial x \partial y}-\frac{\partial f}{\partial x} \frac{\partial^{2} f}{\partial y^{2}}\right) \\
\left(1+\frac{K}{2}\right) \frac{\partial^{2} h}{\partial y^{2}}+f \frac{\partial h}{\partial y}-\frac{\partial f}{\partial y} h-K\left(2 h+\frac{\partial^{2} f}{\partial y^{2}}\right)= \\
x\left(\frac{\partial f}{\partial y} \frac{\partial h}{\partial x}-\frac{\partial f}{\partial x} \frac{\partial h}{\partial y}\right) \\
\frac{1}{\operatorname{Pr}}\left(1+\frac{4}{3} R\right) \frac{\partial^{2} \theta}{\partial y^{2}}+f \frac{\partial \theta}{\partial y}=x\left(\frac{\partial f}{\partial y} \frac{\partial \theta}{\partial x}-\frac{\partial f}{\partial x} \frac{\partial \theta}{\partial y}\right)
\end{array}
$$

subject to the boundary conditions

$$
\begin{gathered}
f=\frac{\partial f}{\partial y}=0, \quad \theta=1, \quad h=-\frac{1}{2} \frac{\partial^{2} f}{\partial y^{2}} \text { at } y=0, \\
\frac{\partial f}{\partial y} \rightarrow 0, \quad \theta \rightarrow 0, \quad h \rightarrow 0 \text { as } y \rightarrow \infty,
\end{gathered}
$$

It can be seen that at the lower stagnation point of the cylinder, $x \approx 0$, equations (16) to (18) reduce to the following nonlinear system of ordinary differential equations:

$$
\begin{gathered}
\left(1+K+\frac{1}{\beta}\right) f^{\prime \prime \prime}+f f^{\prime \prime}-f^{\prime 2}+\theta-M f^{\prime}+K h^{\prime}=0 \\
\left(1+\frac{K}{2}\right) h^{\prime \prime}+f h^{\prime}-f^{\prime} h-K\left(2 h+f^{\prime \prime}\right)=0 \\
\frac{1}{\operatorname{Pr}}\left(1+\frac{4}{3} R\right) \theta^{\prime \prime}+f \theta^{\prime}=0
\end{gathered}
$$

the boundary conditions (19) become

$$
\begin{gathered}
f(0)=f^{\prime}(0)=0, \quad \theta(0)=1, \quad h(0)=-\frac{1}{2} f^{\prime \prime}(0), \\
f^{\prime} \rightarrow 0, \quad \theta \rightarrow 0, \quad h \rightarrow 0 \text { as } y \rightarrow \infty,
\end{gathered}
$$

where primes denote differentiation with respect to $y$.

The physical quantities of interest in this problem are the local skin friction coefficient $C_{f}$ and the local Nusselt number $N_{u}$, and they can be written as

$$
C_{f}=\frac{a^{2}}{\sqrt[4]{G r^{3}} \mu v} \tau_{w}, \quad N_{u}=\frac{a}{\sqrt[4]{G r} k\left(T_{w}-T_{\infty}\right)} q_{w}
$$

where

$$
\tau_{w}=\left(\mu+\frac{\kappa}{2}+\frac{P_{y}}{\sqrt{2 \pi_{c}}}\right)\left(\frac{\partial \bar{u}}{\partial \bar{y}}\right)_{\bar{y}=0}, q_{w}=-k\left(\frac{\partial T}{\partial \bar{y}}\right)_{\bar{y}=0}+q_{r},
$$

using the non-dimensional variables (6)-(8) and the boundary conditions (13) the local skin friction coefficient $C_{f}$ and the local Nusselt number $N_{u}$ are

$$
C_{f}=\left(1+\frac{K}{2}+\frac{1}{\beta}\right) x\left(\frac{\partial^{2} f}{\partial y^{2}}\right)_{y=o}, N_{u}=-\left(1+\frac{4}{3} R\right)\left(\frac{\partial \theta}{\partial y}\right)_{y=o}
$$

\section{SOLUTION PROCEDURES}

Equations (16) to (18) subject to boundary conditions (19) are solved numerically using the Keller-box method as described in the book by Cebeci and Bradshaw Cebeci and Bradshaw (1984). The solution is obtained by the following four steps:

- reduce (16) to (18) to a first-order system,

- write the difference equations using central differences,

- linearize the resulting algebraic equations by Newton's method, and write them in the matrix-vector form,

- solve the linear system by the block tridiagonal elimination technique.

The details of this method can be found in Nazar et al. (2002)

\section{RESULTS AND DISCUSSION}

The numerical solutions of the nonlinear system of partial differential equations (16) to (18) with boundary conditions (19) are solved by the Keller-box method (KBM) with four parameters considered, namely the Prandtl number $\operatorname{Pr}$, the magnetic parameter $M$, the micropolar parameter $K$ and Casson parameter $\beta$. This method is an implicit finitedifference method in conjunction with Newton's method for linearization. This is a suitable method to solve parabolic partial

\begin{tabular}{|c|c|c|c|c|c|c|}
\hline$K$ & & 0 & & & 3 & \\
\hline$x$ & $\begin{array}{c}\text { Merkin } \\
(1976)\end{array}$ & $\begin{array}{c}\text { Nazar } \\
\text { et al. } \\
(2002)\end{array}$ & Present & $\begin{array}{c}\text { Merkin } \\
(1976)\end{array}$ & $\begin{array}{c}\text { Nazar } \\
\text { et al. } \\
(2002)\end{array}$ & Present \\
\hline $0^{\circ}$ & 0.4214 & 0.4214 & 0.421402 & - & 0.3447 & 0.344651 \\
\hline$\pi / 6$ & 0.4161 & 0.4161 & 0.416098 & - & 0.3404 & 0.340350 \\
\hline$\pi / 3$ & 0.4007 & 0.4005 & 0.400607 & - & 0.3277 & 0.327720 \\
\hline$\pi / 2$ & 0.3745 & 0.3741 & 0.374245 & - & 0.3060 & 0.305891 \\
\hline $2 \pi / 3$ & 0.3364 & 0.3355 & 0.335891 & - & 0.2744 & 0.274221 \\
\hline $5 \pi / 6$ & 0.2825 & 0.2811 & 0.281925 & - & 0.2290 & 0.228825 \\
\hline$\pi$ & 0.1945 & 0.1916 & 0.192101 & - & 0.1507 & 0.150645 \\
\hline
\end{tabular}
differential equations. The boundary layer thickness $y_{\infty}=16$ and step size $\Delta y=0.01, \Delta x=0.005$ are used in obtaining the numerical results. The numerical solutions start at the lower stagnation point of the cylinder $x \approx 0$, with initial profiles as given by equations $(20)$ to $(22)$ and proceed round the cylinder up to $x=\pi$.

In order to verify the accuracy of the present applied numerical scheme, a comparison with previously published results has been made. It is noticed from Table 1 that when $\operatorname{Pr}=7, K=0,2, M=0$ and $\beta \rightarrow \infty$, the results under consideration for local Nusselt number $N_{u}$ reduce to the results reported by Merkin (1976) and Nazar et al. (2002) for the case of viscous and micropolar fluids respectively. It is found that the results are a good agreement. Furthermore I believe that Kellerbox method is proven to be very efficient to solve this problems.

Table 1: Comparison of numerical values for the local Nusselt number $N_{u}$ at $\operatorname{Pr}=1, K=0,3, M=0, R=0$ and $\beta \rightarrow \infty$, for viscous value $x$

with previously published results of viscous and micropolar fluid

Table 2 document results for the influence of the microplar parameter $K$ and Casson parameter $\beta$ on heat transfer coefficient and the skin friction coefficient at the lower stagnation point of the cylinder when $\operatorname{Pr}=7, M=1$ and $R=1$. It is observed that, increasing microplar parameter $K$, decreases both skin friction and heat transfer rate. Furthermore, an increase in Casson parameter $\beta$ increases both skin friction and heat transfer rate.

Figures 2-3 illustrates the influence of the Casson parameter $\beta$ on the local Nusselt number and the local skin friction, respectively. It is seen from these figures that an increasing of the Casson parameter leads 
to increases on the local Nusselt number and decreases on the local skin friction. Moreover, as the values of $x$ increase, the rate values of the local Nusselt number decreases and the local skin friction increases.

The behavior of magnetic parameter $M$ on local Nusselt number $N_{u}$ and local skin friction $C_{f}$ are seen in Figures 4-5. It is observed that the local Nusselt number and the local skin friction are increased with the decrease in $M$. Intense amount of magnetic field inside the boundary layer literally increase the Lorentz force which significantly opposes the flow in the reverse direction. Thus, local skin friction coefficient and local Nusselt number rate diminishes

Figures 6-7 show the influence of radiation parameter $R$ local Nusselt number $N_{u}$ and local skin friction $C_{f}$. An increase in $R$ from 0 (non-Radiation case) to 3 , strongly accelerates the flow i.e., increasing in a local skin friction coefficient and local Nusselt number values.

Table 2: The heat transfer coefficient $-(\partial \theta / \partial y)(x, 0)$ and the skin friction coefficient $\left(\partial^{2} f / \partial y^{2}\right)(x, 0)$ at the lower stagnation point of the cylinder, $x \approx 0$, for various values of Casson parameter $\beta$ when $\operatorname{Pr}=7, K=1,3, M=1$ and $R=1$.

\begin{tabular}{|c|c|c|c|c|}
\hline \multirow{2}{*}{$\begin{array}{l}K \\
\beta\end{array}$} & \multicolumn{2}{|c|}{1} & \multicolumn{2}{|c|}{3} \\
\hline & $-\overline{(\partial \theta / \partial y)}$ & $\left(\partial^{2} f / \partial y^{2}\right)$ & $\overline{-(\partial \theta / \partial y)}$ & $\left(\partial^{2} f / \partial y^{2}\right.$ \\
\hline 0.1 & 0.777033 & 0.106734 & 0.762074 & 0.098994 \\
\hline 0.2 & 0.869585 & 0.155168 & 0.839868 & 0.134837 \\
\hline 0.3 & 0.918901 & 0.187037 & 0.878050 & 0.155381 \\
\hline 0.4 & 0.950555 & 0.210040 & 0.901190 & 0.168848 \\
\hline 0.5 & 0.972859 & 0.227552 & 0.916815 & 0.178388 \\
\hline 0.6 & 0.989516 & 0.241376 & 0.928104 & 0.185511 \\
\hline 0.7 & 1.002469 & 0.252587 & 0.936655 & 0.191037 \\
\hline 0.8 & 1.012848 & 0.261873 & 0.943362 & 0.195451 \\
\hline 0.9 & 1.021362 & 0.269696 & 0.948765 & 0.199059 \\
\hline 1.0 & 1.028477 & 0.276380 & 0.953213 & 0.202063 \\
\hline & 1.6 & & & $M=3, R=3, P r=7$ \\
\hline & $\Sigma_{0.8}$ & 030 & & \\
\hline & 赑 & & & \\
\hline
\end{tabular}

Fig. 2 Effect of Casson parameter $\beta$ on local Nusselt number

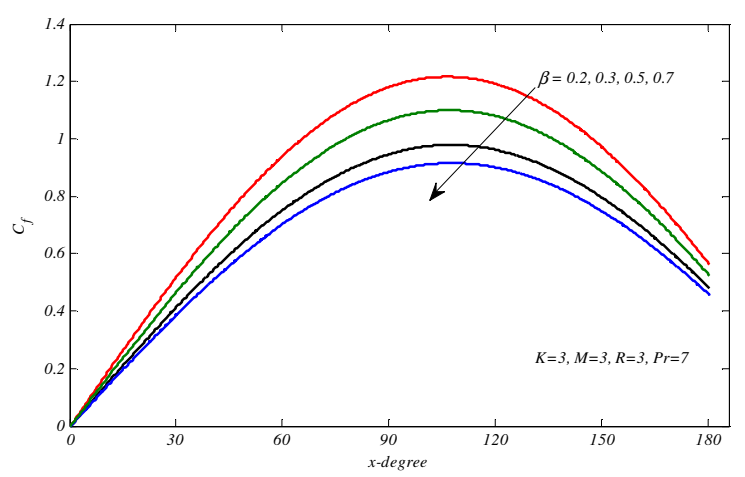

Fig. 3 Effect of Casson parameter $\beta$ on local skin friction coefficient.

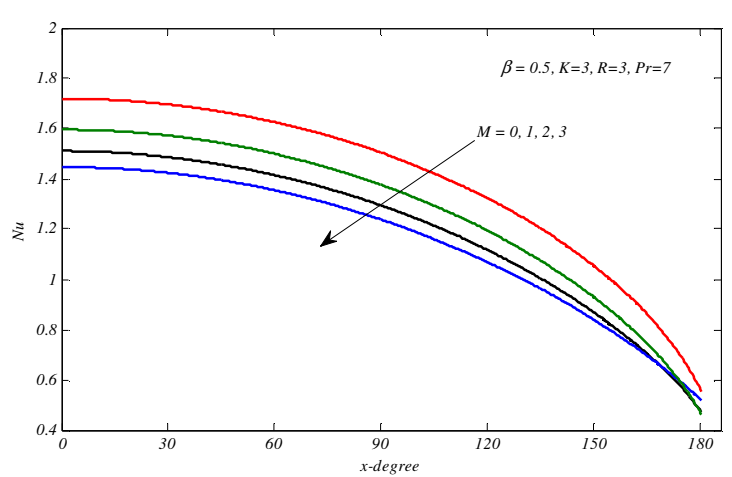

Fig. 4 Effect of magnetic parameter $M$ on local Nusselt number

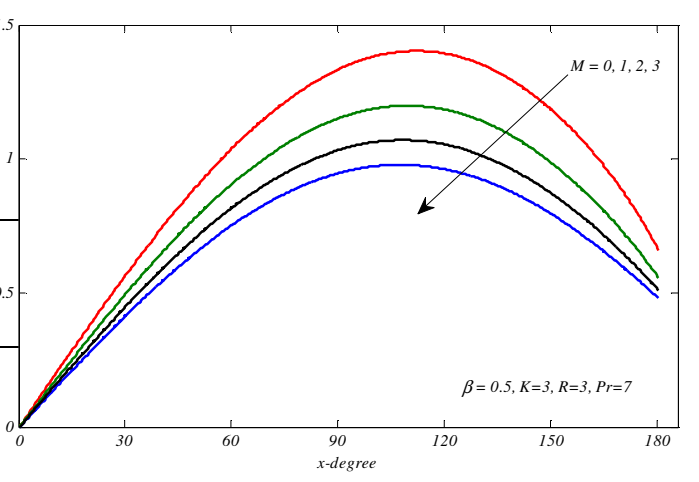

Fig. 5 Effect of magnetic parameter $M$ on local skin friction coefficient.

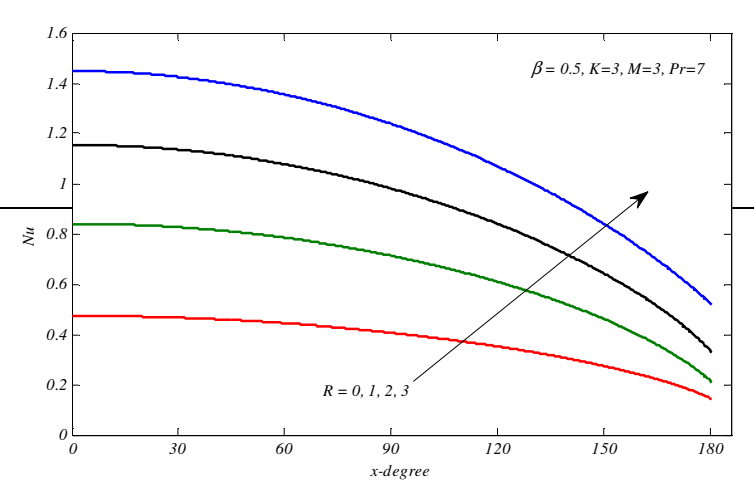

Fig. 6 Effect of radiation parameter $R$ on local Nusselt number

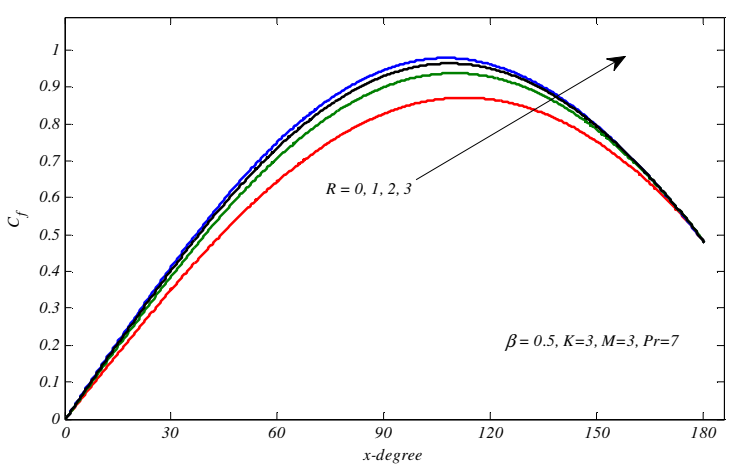

Fig. 7 Effect of radiation parameter $R$ on local skin friction coefficient.

In all profiles a peak arises near the surface of the cylinder and this peak is displaced progressively closer to the wall with an elevation in $R$ values The effect of Casson parameter $\beta$ on temperature, velocity and angular velocity profiles are exhibited in Figures 8 -10. From figure 8 
that the temperature profiles $\theta(0, y)$ increases as decreases the values of $\beta$.

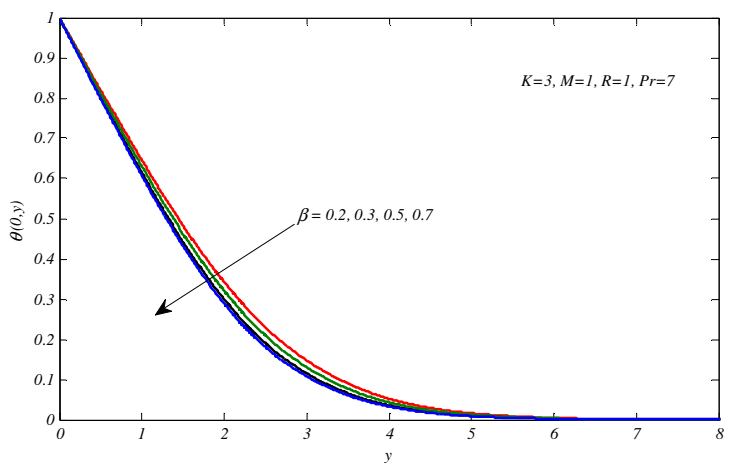

Fig. 8 Effect of Casson parameter $\beta$ on the temperature profiles at the lower stagnation point

Figure. 9 indicates that an increase in $\beta$ tends to decrease in the velocity profiles $(\partial f / \partial y)(0, y)$. It is true because is appeared in the shear term of the momentum equation (15) and an increase in implies a decrease in yield stress of the Casson fluid. Figure 10 shows that as Casson parameter $\beta$ increases, the angular velocity profiles $h(0, y)$ also increases. Physically, an increase in Casson parameter means a decrease in yield stress and increases the plastic dynamic viscosity of the fluid, which makes the momentum boundary layer thicker. This effectively slows down the fluid motion. Figures 11-13 illustrate the effects of several of magnetic parameter $M$ on temperature, velocity and angular velocity profiles. The numerical results obtained show that an increase in the magnetic parameter $M$ the values of temperature profiles $\theta(0, y)$ increases but values of the velocity $(\partial f / \partial y)(0, y)$ and the angular velocity profiles $h(0, y)$ decreases. This is in accordance to the physics of the problem, since the application of a transverse magnetic field results in a resistive type force (Lorentz force) similar to drag force which tends to resist the fluid flow and thus reducing its velocity and angular velocity.

Figures 14-16 present the effect of radiation parameter $R$ on temperature, velocity and angular velocity profiles. The observation shows that the temperature, velocity and angular velocity profiles increases with an increase in $\mathrm{R}$ because increase the value of radiation parameter provides more heat to fluid that causes an enhancement in the temperature, velocity, angular velocity profiles and the thickness of thermal boundary layer.

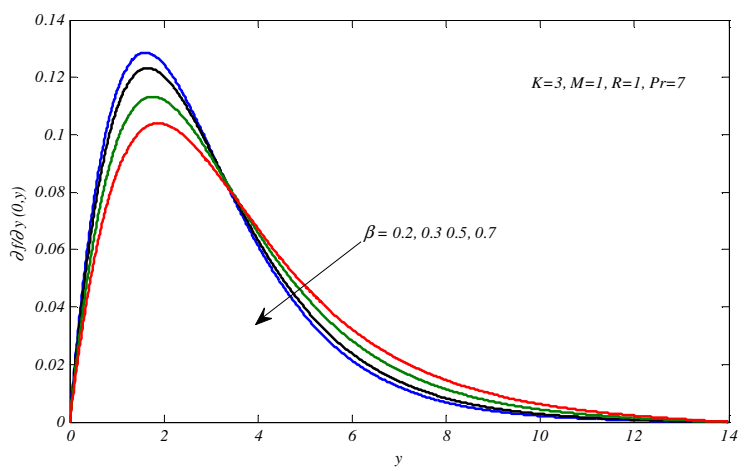

Fig. 9 Effect of Casson parameter $\beta$ on the velocity profiles at the lower stagnation point

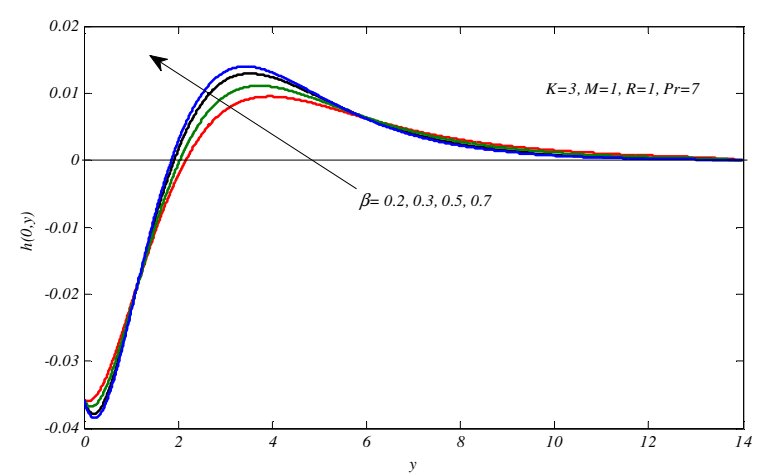

Fig. 10 Effect of Casson parameter $\beta$ on the angular velocity profiles at the lower stagnation point

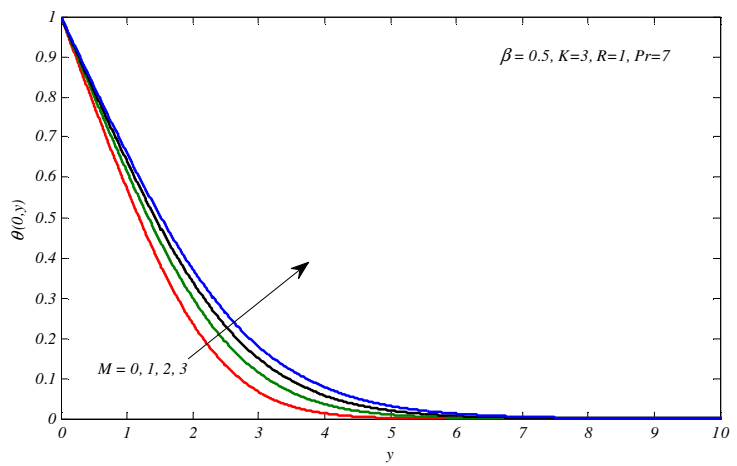

Fig. 11 Effect of magnetic parameter $M$ on the temperature profiles at the lower stagnation point

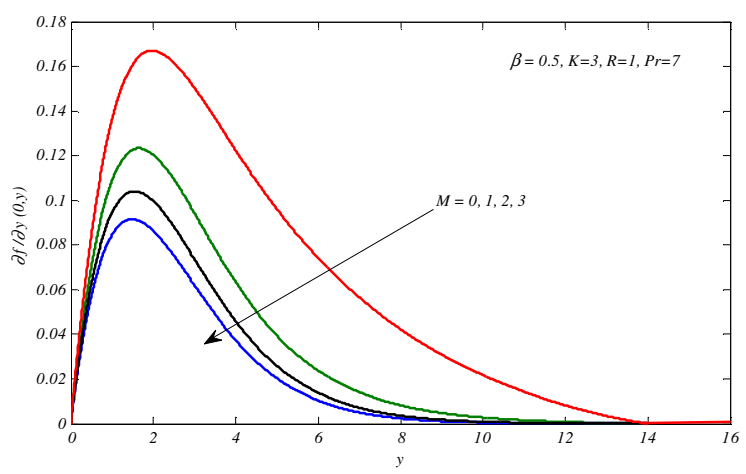

Fig. 12 Effect of magnetic parameter $M$ on the velocity profiles at the lower stagnation point

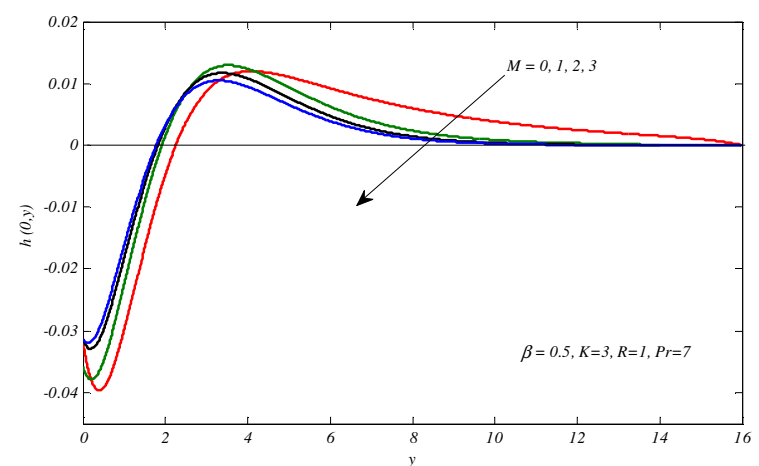

Fig. 13 Effect of magnetic parameter $M$ on the angular velocity profiles at the lower stagnation point 


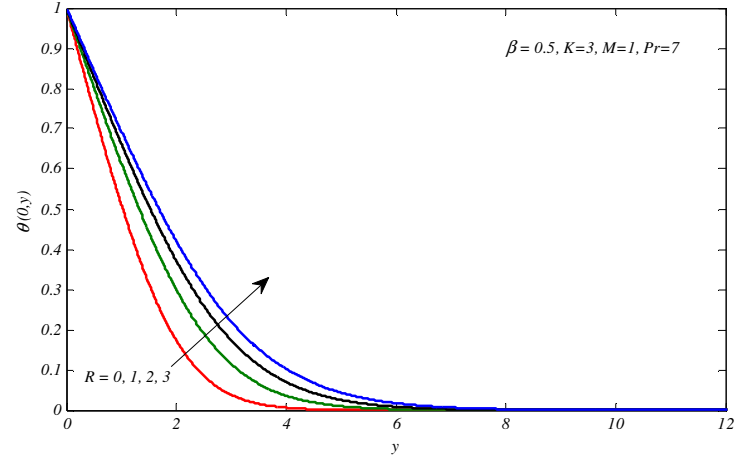

Fig. 14 Effect of radiation parameter $R$ on the temperature profiles at the lower stagnation point

\section{CONCLUSIONS}

In this paper we have theoretically and numerically studied the problem of the effect of MHD free convective boundary layer flow about a cylinder in a micropolar Casson fluid with thermal radiation. We can conclude that, to get a physically acceptable solution:

- With the increase in Casson parameter $\beta$, the local Nusselt number $N_{u}$, the heat transfer coefficient $-(\partial \theta / \partial y)(x, 0)$, the skin friction coefficient $\left(\partial^{2} f / \partial y^{2}\right)(x, 0)$ and velocity profiles $h(0, y)$ increase, while the local skin friction coefficient $C_{f}$, temperature $\theta(0, y)$ and velocity profiles $(\partial f / \partial y)(0, y)$ decrease.

- With the increase in magnetic parameter $M$, the local Nusselt number $N_{u}$, local skin friction coefficient $C_{f}$, velocity $(\partial f / \partial y)(0, y)$ and angular velocity $h(0, y)$ profiles decrease, while the temperature profiles $\theta(0, y)$ increase.

- With the increase in microplar parameter $K$, the skin friction $\left(\partial^{2} f / \partial y^{2}\right)(x, 0)$ and heat transfer $-(\partial \theta / \partial y)(x, 0)$ decrease.

- With the increase in radiation parameter $R$, the local Nusselt number $N_{u}$, local skin friction coefficient $C_{f}$, the temperature $\theta(0, y)$, velocity $(\partial f / \partial y)(0, y)$ and angular velocity $h(0, y)$ profiles increase.

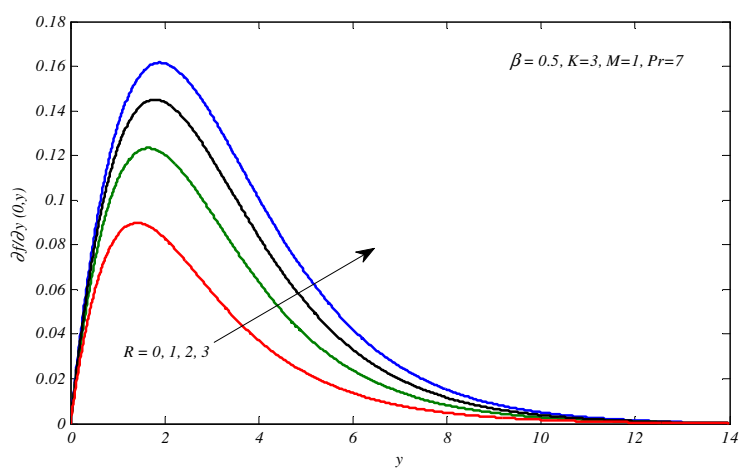

Fig. 15 Effect of radiation parameter $R$ on the velocity profiles at the lower stagnation point

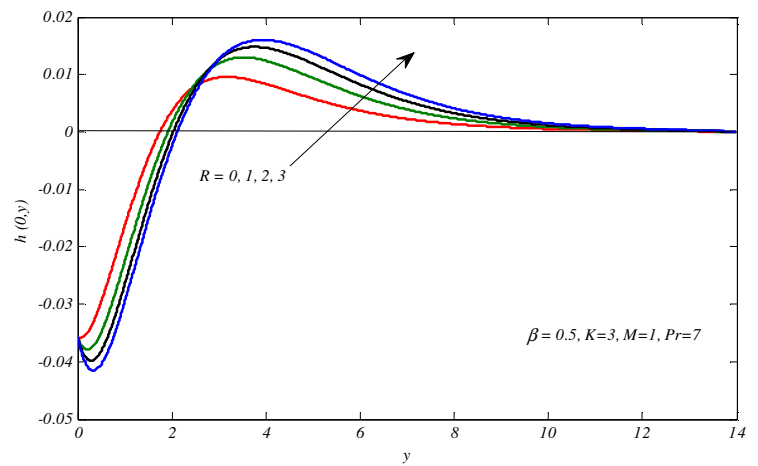

Fig. 16 Effect of radiation parameter $R$ on the angular velocity profiles at the lower stagnation point

\section{ACKNOWLEDGEMENTS}

The author thank to the reviewers for providing valuable comments on this paper.

\section{NOMENCLATURE}

a Radius of the cylinder

$B \quad$ Thermal expansion coefficient

$C_{f} \quad$ Local skin friction coefficient

$c_{\rho} \quad$ Specific heat

$f \quad$ Reduced stream function

$j \quad$ Microinertia density

$\bar{H} \quad$ Angular velocity of micropolar fluid

$g \quad$ Acceleration due to gravity

$\mathrm{Gr} \quad$ Grashof number

$K \quad$ Material or micropolar parameter

$k \quad$ Thermal conductivity

$k^{*} \quad$ Mean absorption coefficient

$M \quad$ Magnetic parameter

$N_{u} \quad$ Local Nusselt number

$R \quad$ Radiation parameter

Pr Prandtl number

$p_{y} \quad$ Yield stress of the fluid

$q_{w} \quad$ Constant wall heat flux

$T \quad$ Fluid temperature

$T_{\infty} \quad$ Temperature of the ambient fluids

$u, v \quad$ Non-dimensional velocity components along $x$ and $y$ directions

$x, y \quad$ Coordinates measured from the lower stagnation point along the surface of cylinder and Normal to it, respectively

Greek Symbols

$\alpha \quad$ Thermal diffusivity

$\beta \quad$ Parameter of the Casson fluid

$\phi \quad$ Spin gradient viscosity

$\theta \quad$ Non-dimensional temperature

$\mu \quad$ Dynamic viscosity

$\mu_{B} \quad$ Plastic dynamic viscosity of the non-Newtonian fluid

$\pi_{c} \quad$ Critical value of this product based on the non-Newtonian model

$\kappa \quad$ Vortex viscosity

$\sigma \quad$ Electric conductivity 


$\begin{array}{ll}\sigma^{*} & \text { Stefan-Boltzmann constant } \\ \alpha & \text { Thermal diffusivity } \\ \rho & \text { Fluid density } \\ v & \text { Kinematic viscosity } \\ \psi & \text { Non-dimensional stream function }\end{array}$

\section{REFERENCES}

Ariman, T., Turk, M., and Sylvester, N., 1973, "Microcontinuum Fluid Mechanics a Review," International Journal of Engineering Science, 11(8), 905-30. https://doi.org/10.1016/0020-7225(73)90038-4

Alkasasbeh, H. T, Sarif, N. M., Salleh, M. Z., Tahar, R. M., Nazar, R., and Pop, I., 2015, "Effect of Radiation And Magnetohydrodynamic Free Convection Boundary Layer Flow on a Solid Sphere with Newtonian Heating in a Micropolar Fluid," AIP Conference Proceedings. 1643(1), 662-669. https://doi.org/10.1063/1.4907509

Alkasasbeh, H. T., Salleh, M. Z., Nazar, R., and Pop, I., 2014, "Numerical Solutions of Radiation Effect on Magnetohydrodynamic Free Convection Boundary Layer Flow about a Solid Sphere with Newtonian Heating," Applied Mathematical Sciences 8(140), 69897000. http://dx.doi.org/10.12988/ams.2014.48649

Bataller, R. C., 2008, "Radiation Effects in the Blasius Flow," Applied Mathematics and Computation, 198(1), 333-38. https://doi.org/10.1016/j.amc.2007.08.037

Blasius, H., 1908, Grenzschichten in Flüssigkeiten mit kleiner Reibung Zeitschrift f urangewandte Mathematik und Physik.

Casson, N., 1959, A Flow Equation for Pigment-oil Suspensions of the Printing Ink Type. Rheology of disperse systems (Editor: Mill, C C), Pergamon, London.

Cebeci, T., and Bradshaw, P., 1984, Physical and Computational Aspects of Convective Heat Transfer. Springer Science \& Business Media

Eringen, A. C., 1966, "Theory of Micropolar Fluids," Journal of Mathematics and Mechanics, 16(1), 1-18.

Eringen, A. C., 2001, Microcontinuum Field Theories: II. Fluent media (Springer).

Gaffar, S. A., Prasad, V. R., and Bég, O. A., 2015, "Computational Analysis of Magnetohydrodynamic Free Convection Flow and Heat Transfer of Non-newtonian Tangent Hyperbolic Fluid From a Horizontal Circular Cylinder with Partial Slip," International Journal of Applied and Computational Mathematics, 1(4), 651-75. https://doi.org/10.1007/s40819-015-0042-x

Gaffar, S. A., Prasad, V. R., and Reddy, E. K., 2017, "Magnetohydrodynamic Free Convection Flow and Heat Transfer of Non-newtonian Tangent Hyperbolic Fluid From Horizontal Circular Cylinder with Biot Number Effects," International Journal of Applied and Computational Mathematics, 3(2), 721-43. https://doi.org/10.1007/s40819-015-0130-y

Haq, R., Nadeem, S., Khan, Z., and Okedayo, T., 2014, "Convective Heat Transfer and MHD Effects on Casson Nanofluid Flow over a Shrinking Sheet," Open Physics, 12(12), 862-71. https://doi.org/10.2478/s11534-014-0522-3

Hussanan, A., Salleh, M. Z., Tahar, R. M., and Khan, I., 2014, "Unsteady Boundary Layer Flow and Heat Transfer of a Casson Fluid Past an Oscillating Vertical Plate with Newtonian Heating," PLoS ONE, 9(10), 1-9. https://doi.org/10.1371/journal.pone.0108763

Ingham, D., 1978, "Free-convection Boundary Layer on an Isothermal Horizontal Cylinder," Zeitschrift für Angewandte Mathematik und Physik (ZAMP), 29(6), 871-83. https://doi.org/10.1007/BF01590813
Khalid, A., Khan, I., Khan, A., and Shafie, S., 2015, "Unsteady MHD Free Convection Flow of Casson Fluid Past over an Oscillating Vertical Plate Embedded in a Porous Medium," Engineering Science and Technology, an International Journal, 18(3), 309-17. https://doi.org/10.1016/i.jestch.2014.12.006

Lukaszewicz, G., 1999, Micropolar fluids: theory and applications Springer Science \& Business Media.

Mahdy, A., and Ahmed, S. E., 2017, "Unsteady MHD Convective Flow of Non-Newtonian Casson Fluid in the Stagnation Region of an Impulsively Rotating Sphere," Journal of Aerospace Engineering, 30(5), $\quad 04017036 . \quad$ https://doi.org/10.1061/(ASCE)AS.19435525.0000700

Mehmood, Z., Mehmood, R., and Iqbal, Z., 2017, "Numerical Investigation of Micropolar Casson Fluid over a Stretching Sheet with Internal Heating," Communications in Theoretical Physics, 67(4), 44348. https://doi.org/10.1088/0253-6102/67/4/443

Merkin, J., D., 1976, "Free Convection Boundary Layer on an Isothermal Horizontal Cylinder," ASME/AIChe Heat Transfer Conference, St. Louis, USA. https://doi.org/10.1007/BF01590813

Merkin, J., and Pop, I., 1988, "A Note on the Free Convection Boundary Layer on a Horizontal Circular Cylinder With Constant Heat Flux," Wärme-und Stoffübertragung, 22(1-2), 79-81. https://doi.org/10.1007/BF01001575

Mukhopadhyay, S., Bhattacharyya, K., and Hayat, T., 2013, "Exact Solutions for the Flow of Casson Fluid over a Stretching Surface with Transpiration and Heat Transfer Effects," Chinese Physics B, 22(11), 16. https://doi.org/10.1088/1674-1056/22/11/114701

Mustafa, M., Hayat, T., Pop, I., and Aziz, A., 2011, "Unsteady Boundary Layer Flow of a Casson Fluid due to an Impulsively Started Moving Flat Plate," Heat Transfer Asian Research, 40(6), 563-76. https://doi.org/10.1002/htj.20358

Nagendra, N., Amanulla, C., Narayana Reddy, M., Rao, A., and Bég, O., 2017, "Mathematical Study of Non-Newtonian Nanofluid Transport Phenomena from an Isothermal Sphere," Frontiers in Heat and Mass Transfer, 8(29), 1-13. http://dx.doi.org/10.5098/hmt.8.29

Nazar, R., Amin, N., and Pop, I., 2002, "Free Convection Boundary Layer on an Isothermal Horizontal Circular Cylinder in a Micropolar Fluid," Heat Transfer, 2, 525-30.

Qasim, M., and Noreen, S., 2014, "Heat Transfer in the Boundary Layer Flow of a Casson Fluid over a Permeable Shrinking Sheet with Viscous Dissipation," The European Physical Journal Plus, 129(1), 1-8. https://doi.org/10.1140/epjp/i2014-14007-5

Ramachandra, P., V, Rao, A. S., and Bég, O. A., 2013, "Flow and Heat Transfer of Casson Fluid from a Horizontal Circular Cylinder with Partial Slip in Non-darcy Porous Medium," Journal of Applied and Computational Mathematics, 2(127), 2-12. http://dx.doi.org/10.4172/2168-9679.1000127

Salleh, M. Z., and Nazar, R., 2010, "Free Convection Boundary Layer Flow over a Horizontal Circular Cylinder with Newtonian Heating," Sains Malaysiana, 39(4), 671-76.

Shehzad, S., Hayat, T., Qasim, M., and Asghar, S., 2013, "Effects of Mass Transfer on MHD Flow of Casson Fluid with Chemical Reaction and Suction," Brazilian Journal of Chemical Engineering, 30(1), 18795. http://dx.doi.org/10.1590/S0104-66322013000100020

Subba, R., A, Ramachandra Prasad, V., Bhaskar Reddy, N., and Anwar Bég, O., 2015, "Heat Transfer in a Casson Rheological Fluid from a Semi-infinite Vertical Plate with Partial Slip," Heat Transfer Asian Research, 44(3), 272-91. https://doi.org/10.1002/htj.21115 
Frontiers in Heat and Mass Transfer (FHMT), 10, 32 (2018)

Global Digital Central DOI: 10.5098/hmt.10.32

ISSN: 2151-8629 